\title{
ON THE BOUNDARY BEHAVIOR OF A CONFORMAL MAP
}

BY

\section{J. L. WALSH AND L. ROSENFELD}

The object of this paper is to indicate the immediate usefulness of Caratheodory's theory of the conformal mapping of variable regions in the study of boundary behavior of a fixed but arbitrary conformal map. We study especially the mapping of an infinite strip and its behavior at infinity.

Studies of an infinite strip by other methods have previously been made, especially by Ahlfors, Ferrand, Ostrowski, and Warschawski; for a résumé see Gattegno and Ostrowski [6]. The present results are more directly geometric than these previous ones in both method and conclusion; broadly speaking, they are in some respects more and in other respects less general than the previous ones.

The theory of conformal mapping of variable regions introduced by Carathéodory in 1912 was employed by Montel in 1917 to study the properties of prime ends under conformal mapping. That theory has more recently been used in the study of boundary behavior of conformal maps by Ferrand $[7 ; 8]$, emphasized by Walsh [2], and used for the study of strips by Madame Lelong-Ferrand [3]. The essential difference between the latter and the present paper is that here we consistently use both translation and stretching of the original region to obtain a sequence of variable regions possessing a kernel, whereas Madame Lelong-Ferrand uses primarily translation. The present results are thus more general, both where the width of the strip (the variable $2 \phi(u)$, in the notation of $\$ 2$ below) has an infinite number of limit values, and more especially where that limit is zero or infinite.

We introduce a new condition (property $\mathrm{B}$, below) on the boundary of an infinite strip, which is useful in the study of conformal mapping of the strip. The intrinsic properties of this condition are studied in $\$ 1$, and applications to conformal mapping in successively more general situations are considered in $\$ \S 2-5$. The extension of property $B$ and its implications for finite boundary points are studied in $\$ 6$, and the relation of property $B$ to various other conditions on the boundary of an infinite strip is investigated in $\$ 7$.

Property B is exhibited as a sufficient (but not necessary) condition for the fundamental asymptotic relations, such as (2.1), a less restrictive condition than that for an $L$-strip.

1. Property B. If $\phi(u)$ is a real function of the real variable $u$ defined for

Presented to the Society, December 27, 1951 and April 25, 1953; received by the editors October 28, 1953. 
$u_{1} \leqq u<+\infty\left(-\infty \leqq u_{1}<+\infty\right)$, and if we have uniformly in every interval $I$ : $|U| \leqq U_{0}$ the equation

$$
\lim _{u \rightarrow+\infty} \frac{\phi[U \phi(u)+u]}{\phi(u)}=1,
$$

then $\phi(u)$ is said to have property B (at infinity). We do not require that $\phi(u)$ be single-valued, but do require that (1.1) hold for any choice of values of $\phi(u)$. It will appear from our use of $(1.1)$ that the same value $\phi(u)$ should be used in the denominator and inside the square bracket in the numerator. But an arbitrary value of $\phi[U \phi(u)+u]$ may be used, even in the case $U=0$. In particular, we deduce

$$
\lim _{u \rightarrow+\infty} \frac{\max \phi(u)}{\min \phi(u)}=1,
$$

where the maximum and minimum are taken over $\phi(u)$ for each fixed $u$.

When we add (or subtract) two multiple-valued functions of this kind, we add (or subtract) all functional values for each value of the independent variable.

Property B is the fundamental requirement that we impose on the boundary of an infinite strip. We now develop for reference some easily proved intrinsic consequences of the definition, without reference to conformal mapping. We retain the notation used in the definition.

THEOREM 1.1. If $\phi(u)$ has property B at infinity, and if $\alpha(u, t)$ defined for $u>u_{1}, t_{0} \leqq t \leqq t_{1}\left(-\infty \leqq t_{0}<t_{1} \leqq \infty\right)$, has the property

$$
\left|\frac{\alpha(u, t)-u}{\phi(u)}\right| \leqq M, \quad u \geqq u_{1}, t_{0} \leqq t \leqq t_{1},
$$

then we have

$$
\lim _{u \rightarrow+\infty} \frac{\phi[\alpha(u, t)]}{\phi(u)}=1
$$

uniformly for all $t, t_{0} \leqq t \leqq t_{1}$.

We set

$$
\alpha(u, t) \equiv B(u, t) \phi(u)+u,
$$

whence $|B(u, t)| \leqq M$ for $u \geqq u_{1}, t_{0} \leqq t \leqq t_{1}$. Thus we have

$$
\frac{\phi[\alpha(u, t)]}{\phi(u)} \equiv \frac{\phi[B(u, t) \phi(u)+u]}{\phi(u)},
$$

and the theorem follows from (1.1).

The proof of Theorem 1.2 is similar to the proof just given and is omitted: 
THEOREM 1.2. If $\phi(u)$ has property B at infinity, and if $u_{n} \rightarrow+\infty, u_{n}^{\prime} \rightarrow+\infty$ with

$$
\limsup _{n \rightarrow \infty}\left|\frac{u_{n}-u_{n}^{\prime}}{\phi\left(u_{n}\right)}\right|<\infty,
$$

then we have

$$
\lim _{n \rightarrow \infty} \frac{\phi\left(u_{n}\right)}{\phi\left(u_{n}^{\prime}\right)}=1
$$

It can be verified immediately that the function $\phi(u) \equiv u^{1 / 2}$ has property $\mathrm{B}$ at infinity. Nevertheless, that property limits the order of $\phi(u)$ as $u \rightarrow+\infty$ :

TheOREM 1.3. If $\phi(u)$ has property B at infinity, then

$$
\lim _{u \rightarrow \infty} \phi(u) / u=0 \text {. }
$$

If this conclusion is false, there exists a sequence $u_{n} \rightarrow \infty$ such that

$$
\lim _{n \rightarrow \infty} \frac{u_{n}}{\phi\left(u_{n}\right)}=\lambda
$$

with $|\lambda|<\infty$. Of course we have $\phi\left(u_{n}\right) \rightarrow+\infty$, whence by (1.4) and (1.1)

$$
\lim _{n \rightarrow \infty} \frac{\phi\left[\left(\left(a-u_{n}\right) / \phi\left(u_{n}\right)\right) \phi\left(u_{n}\right)+u_{n}\right]}{\phi\left(u_{n}\right)}=1,
$$

where the constant $a$ is arbitrary. The numerator in the first member of (1.5) reduces to $\phi(a)$, so $\phi(u)$ is identically constant (not zero, by (1.1)), in contradiction to (1.4).

TheOREM 1.4. If $\phi(u)$ has property B at infinity and if $\psi(u)$ is a real function of $u$ defined for $u \geqq u_{1}$, which satisfies

$$
\lim _{u \rightarrow+\infty} \psi(u) / \phi(u)=1,
$$

then $\psi(u)$ has property B at infinity.

It follows from (1.6) and from Theorem 1.3 that $\psi(u) / u \rightarrow 0$ as $u \rightarrow \infty$. Then for $U$ in $I:|U| \leqq U_{0}$ we have uniformly

$$
U \psi(u)+u=u\left(U \frac{\psi(u)}{u}+1\right) \rightarrow+\infty .
$$

We write

$$
\frac{\psi[U \psi(u)+u]}{\psi(u)}=\frac{\psi[U \psi(u)+u]}{\phi[U \psi(u)+u]} \frac{\phi[U(\psi(u) / \phi(u)) \phi(u)+u]}{\phi(u)} \frac{\phi(u)}{\psi(u)} ;
$$


the first factor in the second member approaches unity as $u \rightarrow+\infty$ by (1.6) because the common argument of $\psi$ and $\phi$ becomes infinite. Thus each factor in the second member of (1.7) approaches unity uniformly for $U$ in $I$, and the theorem is established.

It is clear from (1.1) that if $\phi(u)$ has property B at infinity and if the constant $\lambda(\neq 0)$ is arbitrary, then $\lambda \phi(u)$ has property B at infinity. From this remark and Theorem 1.4 we have

THEOREM 1.5. Theorem 1.4 remains valid if (1.6) is replaced by

$$
\lim _{u \rightarrow+\infty} \psi(u) / \phi(u)=\lambda \neq 0 .
$$

Theorem 1.5 cannot be strengthened merely by replacing (1.8) by

$$
0<a \leqq\left|\frac{\phi(u)}{\psi(u)}\right| \leqq b<\infty, \quad u \geqq u_{1},
$$

as the reader may show from the counter-example $\phi(u) \equiv 1, \psi(u) \equiv 1+2^{-1} \sin u$.

Property B persists under addition, if an auxiliary condition is provided:

THEOREM 1.6. If $\phi_{1}(u)$ and $\phi_{2}(u)$ have property $\mathrm{B}$ at infinity, and if we have for $u \geqq u_{1}$

$$
0<a \leqq \phi_{1}(u) / \phi_{2}(u) \leqq b<\infty,
$$

then $\phi(u) \equiv \phi_{1}(u)+\phi_{2}(u)$ has property $\mathrm{B}$ at infinity.

Theorem 1.6 follows from the identity

$$
\begin{aligned}
\frac{\phi[U \phi(u)+u]}{\phi(u)}-1 & \equiv \frac{\left[\alpha_{1}(u)-1\right] \phi_{1}(u) / \phi_{2}(u)+\alpha_{2}(u)-1}{1+\phi_{1}(u) / \phi_{2}(u)}, \\
\alpha_{1}(u) & \equiv \frac{\phi_{1}\left[\left(U+U \phi_{2}(u) / \phi_{1}(u)\right) \phi_{1}(u)+u\right]}{\phi_{1}(u)}, \\
\alpha_{2}(u) & \equiv \frac{\phi_{2}\left[\left(U+U \phi_{1}(u) / \phi_{2}(u)\right) \phi_{2}(u)+u\right]}{\phi_{2}(u)},
\end{aligned}
$$

where $\alpha_{1}(u) \rightarrow 1$ and $\alpha_{2}(u) \rightarrow 1$ uniformly for $U$ in $I$.

Since we have not required singlevaluedness of a function in order that it have property $\mathrm{B}$, it may occur in Theorem 1.6 that the locus $v=\phi_{1}(u)+\phi_{2}(u)$ separates the plane even when neither of the loci $v=\phi_{1}(u)$ and $v=\phi_{2}(u)$ separates the plane. For instance, suppose in the interval $I_{0}:|u|<2$ the function $\phi_{1}(u)$ consists of $v=1$ together with the segment of the line $v=1+u$, $0 \leqq u \leqq 1$, and $\phi_{2}(u)$ in $I_{0}$ consists of $v=1$ together with the segment of the line $v=2-u, 0 \leqq u \leqq 1$. Then $\phi_{1}(u)+\phi_{2}(u)$ consists of $v=2$, the two line segments $v=2+u$ and $v=3-u, 0 \leqq u \leqq 1$, and also the segment $v=3,0 \leqq u \leqq 1$. This ambiguity leads to no difficulty, by virtue of our convention that such 
relations as (1.1) and (1.9) hold for all choices of functional values.

The locus $v=\phi(u),-\infty \leqq u_{0} \leqq u<+\infty$, is said to have $a$ horizontal $L$ tangent (at infinity) if we have $\left(u_{1} \rightarrow \infty, u_{2} \rightarrow \infty\right)$

$$
\frac{\phi\left(u_{2}\right)-\phi\left(u_{1}\right)}{u_{2}-u_{1}} \rightarrow 0 \text {. }
$$

It follows that $\phi(u) / u \rightarrow 0$ as $u \rightarrow \infty$, for if $u_{n}$ is any sequence $\left(u_{n} \rightarrow \infty\right)$ for which $\phi\left(u_{n}\right) / u_{n}$ approaches a limit finite or infinite we have by (1.10)

$$
0=\lim _{u \rightarrow \infty}\left[\lim _{u_{n} \rightarrow \infty} \frac{\phi\left(u_{n}\right) / u_{n}-\phi(u) / u_{n}}{1-u / u_{n}}\right]=\lim _{u_{n} \rightarrow \infty} \frac{\phi\left(u_{n}\right)}{u_{n}} .
$$

If $v=\phi(u)$ has a horizontal L-tangent at infinity, then $\phi(u)$ has property B at infinity, for with $0<|U|<U_{0}$ and $u_{2}=U \phi\left(u_{1}\right)+u_{1}$ we have by (1.10)

$$
U \frac{\phi\left[U \phi\left(u_{1}\right)+u_{1}\right]-\phi\left(u_{1}\right)}{U \phi\left(u_{1}\right)} \rightarrow 0
$$

uniformly with respect to $U$ since $u_{1} \rightarrow \infty$ implies $u_{2} \rightarrow \infty$ uniformly; of course $U=0$ in $(1.1)$ is trivial.

2. Symmetric infinite strips. We turn now to our main purpose, study of the conformal mapping of infinite strips. Symmetric strips illustrate the method in its simplest form, and are important as a preliminary topic. We postpone detailed references to the literature until the more general results are established.

TheOREM 2.1. Let $S$ be a simply connected region of the $w(=u+i v)$-plane which contains the segment $u_{1} \leqq u$ of the axis of reals and is symmetric in the axis of reals. Let the boundary of $S$ in the half-plane $u_{1} \leqq u$ consist of the two loci $v=\phi(u), v=-\phi(u)$, where $\phi(u) \geqq 0$ and has property B at infinity. Let $w=f(z)$ $\equiv u(z)+i v(z)$ map the infinite strip $\Sigma:|y|<\pi / 2$ of the $z(=x+i y)$-plane onto $S$ in such a way that $f(z)$ is real when $z$ is real and $\lim _{x \rightarrow \infty} u(x+i y)=+\infty$. Then if $x_{n}$ is any sequence of real numbers with $x_{n} \rightarrow+\infty$, we have

$$
\lim _{n \rightarrow \infty} \frac{f\left(z+x_{n}\right)-f\left(x_{n}\right)}{\phi\left[u\left(x_{n}\right)\right]}=\frac{2 z}{\pi}
$$

for $z$ in $\Sigma$, uniformly on any closed bounded subset of $\Sigma$.

We suppose here and in similar cases below that the region $S$ is actually a strip, in the sense that each point $w=u+i v$ of $S$ lies interior to a vertical finite line segment bounded by some point of each of the loci $v= \pm \phi(u)$. The purpose of this hypothesis is to exclude such a region as the $w$-plane slit along the two infinite segments $u \geqq 0, v= \pm 1$; even such a slit region, however, can be treated by our methods with little or no modification.

The function $f(z)$ is uniquely determined except for an arbitrary horizontal 
translation of $\Sigma$, namely $z^{\prime}=z-\alpha$ where $\alpha$ is real. We introduce the notation

$$
f_{n}(z) \equiv u_{n}(z)+i v_{n}(z) \equiv \frac{f\left(z+x_{n}\right)-f\left(x_{n}\right)}{\phi\left[u\left(x_{n}\right)\right]}, \quad w_{n}=f\left(x_{n}\right) .
$$

Since $w=f\left(z+x_{n}\right)$ maps $\Sigma$ onto $S$, the transformation $w^{\prime}=f\left(z+x_{n}\right)-f\left(x_{n}\right)$ maps $\Sigma$ onto $S_{n}^{\prime}$, namely $S$ horizontally translated so that $w_{n}$ in $S$ becomes $w^{\prime}=0$ in $S_{n}^{\prime}$. The denominator in (2.2) has the property of changing the size but not the orientation of $S_{n}^{\prime}$ (leaving the origin fixed) so that the boundary of the new image $S_{n}$ of $\Sigma$ under the transformation $W=U+i V=f_{n}(z)$ passes through the point $W=i$. We prove that the kernel of the regions $S_{n}$ in the sense of Caratheodory consists of the strip $|V|<1$.

If we set $w_{n}=f\left(x_{n}\right)=u\left(x_{n}\right)+i v\left(x_{n}\right)$, the strip $S_{n}^{\prime}$ in the plane of $w^{\prime}=u^{\prime}+i v^{\prime}$ is bounded by the curves whose ordinates are $v^{\prime}= \pm \phi\left[u^{\prime}+u\left(x_{n}\right)\right]$, respectively. We now set $W=w^{\prime} / \phi\left[u\left(x_{n}\right)\right], V=v^{\prime} / \phi\left[u\left(x_{n}\right)\right], U=u^{\prime} / \phi\left[u\left(x_{n}\right)\right]$, so $S_{n}$ is bounded by the curves whose ordinates are

$$
V= \pm \phi\left[U \phi\left[u\left(x_{n}\right)\right]+u\left(x_{n}\right)\right] / \phi\left[u\left(x_{n}\right)\right] .
$$

Since by (1.1) these ordinates approach the respective values $V= \pm 1$ uniformly on every interval $|U| \leqq U_{0}$, it follows that any region $|V|<1-\delta$, $|U|<U_{0}, \delta>0$, lies in all $S_{n}$ for $n$ sufficiently large; but no region containing both $W=0$ and a point exterior to $|V|<1$ lies in all $S_{n}$ for $n$ sufficiently large. Consequently the regions $S_{n}$ converge to the kernel $|V|<1$. Equation (2.1) now follows by Carathéodory's results, for we have $f_{n}(0)=0, f_{n}^{\prime}(0)>0$.

Theorem 2.1 has various applications:

THEOREM 2.2. With the hypothesis of Theorem 2.1 let $z_{n}=x_{n}+i y_{n}$ and $\boldsymbol{z}_{n}^{\prime}=x_{n}^{\prime}+i y_{n}^{\prime}$ be two sequences of points in $\Sigma$ such that $\left|y_{n}\right|<a<\pi / 2,\left|y_{n}^{\prime}\right|<a$ $<\pi / 2,\left|x_{n}-x_{n}^{\prime}\right|<b<\infty$. Then we have

$$
\begin{gathered}
\lim _{n \rightarrow \infty} \frac{\phi\left[u\left(z_{n}^{\prime}\right)\right]}{\phi\left[u\left(z_{n}\right)\right]}=1, \\
\lim _{n \rightarrow \infty}\left[\frac{f\left(z_{n}\right)-f\left(z_{n}^{\prime}\right)}{\phi\left[u\left(z_{n}\right)\right]}-\frac{2}{\pi}\left(z_{n}-z_{n}^{\prime}\right)\right]=0 .
\end{gathered}
$$

Substitution $z=i y_{n}$ in an equation equivalent to (2.1) yields

$$
\lim _{n \rightarrow \infty}\left[\frac{f\left(z_{n}\right)-f\left(x_{n}\right)}{\phi\left[u\left(x_{n}\right)\right]}-\frac{2 i}{\pi} y_{n}\right]=0,
$$

and the corresponding equation for $z_{n}^{\prime}$ is

$$
\lim _{n \rightarrow \infty}\left[\frac{f\left(z_{n}^{\prime}\right)-f\left(x_{n}^{\prime}\right)}{\phi\left[u\left(x_{n}^{\prime}\right)\right]}-\frac{2 i}{\pi} y_{n}^{\prime}\right]=0 ;
$$


substitution $z=x_{n}^{\prime}-x_{n}$ in an equation equivalent to (2.1) yields

$$
\lim _{n \rightarrow \infty}\left[\frac{u\left(x_{n}^{\prime}\right)-u\left(x_{n}\right)}{\phi\left[u\left(x_{n}\right)\right]}-\frac{2}{\pi}\left(x_{n}^{\prime}-x_{n}\right)\right]=0 .
$$

The real part of the first member of (2.5) is

$$
\lim _{n \rightarrow \infty} \frac{u\left(z_{n}\right)-u\left(x_{n}\right)}{\phi\left[u\left(x_{n}\right)\right]}=0 .
$$

Theorem 1.2 in conjunction with (2.8) and (2.7) respectively yields

$$
\lim _{n \rightarrow \infty} \frac{\phi\left[u\left(z_{n}\right)\right]}{\phi\left[u\left(x_{n}\right)\right]}=\lim _{n \rightarrow \infty} \frac{\phi\left[u\left(x_{n}\right)\right]}{\phi\left[u\left(x_{n}^{\prime}\right)\right]}=1,
$$

and we similarly have

$$
\lim _{n \rightarrow \infty} \frac{\phi\left[u\left(z_{n}^{\prime}\right)\right]}{\phi\left[u\left(x_{n}^{\prime}\right)\right]}=1 .
$$

Equation (2.3) is now immediate, and (2.4) is a consequence of (2.5), (2.6), (2.7).

A consequence of (2.1) or (2.4) is especially significant geometrically; in (2.5) we suppose $y_{n} \rightarrow y_{0},\left|y_{0}\right|<\pi / 2$, whence

$$
\lim _{n \rightarrow \infty} \frac{v\left(z_{n}\right)}{\phi\left[u\left(x_{n}\right)\right]}=\frac{2 y_{0}}{\pi} ;
$$

the ratio of the ordinates $y_{n}$ and $\pi / 2$ approaches the same limit as the ratio of the corresponding ordinates in the $w$-plane. By way of a converse, we prove

THEOREM 2.3. With the hypothesis of Theorem 2.1 let $\gamma$ be a curve in $S$ defined for $u>u_{1}$ by the equation $v=\psi(u)$, with $\lim _{u \rightarrow \infty} \psi(u) / \phi(u)=\lambda$. If $\Gamma$ is the image in the z-plane of $\gamma$, we have on $\Gamma$

$$
\lim _{x \rightarrow \infty} y=\frac{\pi \lambda}{2} \text {. }
$$

We suppose first $|\lambda|<1$, and choose $\delta(>0)$ so that $|\lambda \pm \delta|<1$. Denote by $\Gamma_{\lambda+\delta}$ and $\Gamma_{\lambda-\delta}$ the lines $y=\pi(\lambda+\delta) / 2$ and $y=\pi(\lambda-\delta) / 2$, respectively, and by $\gamma_{\lambda+\delta}$ and $\gamma_{\lambda \rightarrow \delta}$ their images in the $w$-plane. By (2.9) we have for $z$ on $\Gamma_{\lambda_{ \pm} \delta}$

$$
\lim _{x \rightarrow \infty} \frac{v(z)}{\phi[u(x)]}=\lambda \pm \delta
$$

respectively. For $u$ sufficiently large it follows from (2.11) that $\gamma$ lies between $\gamma_{\lambda+\delta}$ and $\gamma_{\lambda-\delta}$, so it follows from the topological properties of the conformal map that for $u$ (or $x$ ) sufficiently large, $\Gamma$ lies between $\Gamma_{\lambda+8}$ and $\Gamma_{\lambda \rightarrow}$, which is equivalent to $(2.10)$. 
3. Nonsymmetric strips. Theorem 2.1 is to be regarded as preliminary; we shall prove several analogues under varying hypotheses which apply to strips not necessarily symmetric.

THEOREM 3.1. Let $S$ be an infinite simply connected strip in the $w(=u+i v)$ plane which contains the segment $u>u_{1}, v=0$, and whose boundaries can be represented in the form $v=\phi_{+}(u)$ and $v=\phi_{-}(u)$, with $\phi_{+}(u)>0, \phi_{-}(u)<0$, $\lim _{u \rightarrow \infty} \phi_{-}(u) / \phi_{+}(u)=\lambda$, where $\phi_{+}(u)$ and $\phi_{-}(u)$ are not necessarily single valued. Suppose $\theta(u) \equiv \phi_{+}(u)-\phi_{-}(u)$ has property B at infinity. Let $w=f(z)=u(z)$ $+i v(z)$ map the infinite strip $\Sigma_{z}:|y|<\pi / 2$ onto $S$ so that $\lim _{x \rightarrow \infty} u(z)=+\infty$. Then if $x_{n}$ is any sequence of real points with $x_{n} \rightarrow+\infty$, we have

$$
\lim _{n \rightarrow \infty} \frac{f\left(z+x_{n}\right)-f\left(x_{n}\right)}{\theta\left[u\left(x_{n}\right)\right]}=\frac{z}{\pi}
$$

throughout $\Sigma_{z}$, uniformly on any closed bounded subset of $\Sigma_{z}$.

The case $\lambda=-\infty$ is treated by interchanging the roles of $\phi_{+}(u)$ and $\phi_{-}(u)$, whence we have $\lambda=0$. The case $\lambda=0$ is to be treated separately by special methods later, so for the present we assume $0>\lambda>-\infty$.

From the conditions on $\phi_{+}(u)$ and $\phi_{-}(u)$ we have

$$
\lim _{u \rightarrow+\infty} \frac{\phi_{+}(u)-\phi_{-}(u)}{\phi_{+}(u)}=1-\lambda,
$$

and from Theorem 1.5 it follows that $\phi_{+}(u)$ has property B; similarly it follows that $\phi_{-}(u)$ has property $\mathrm{B}$.

For each $u$ sufficiently large we have $\min \theta(u)>\max \phi_{+}(u)$. Indeed, we note

$$
\frac{\min \theta(u)}{\max \phi_{+}(u)}=\frac{\min \phi_{+}(u)}{\max \phi_{+}(u)}-\frac{\max \phi_{-}(u)}{\max \phi_{+}(u)} ;
$$

the first term of the second member approaches unity and the second term approaches $-\lambda$ as $u$ becomes infinite. Similarly we have for $u$ sufficiently large $\min \theta(u)>-\min \phi_{-}(u)$.

The asymptotic behavior of $f(z)$ depends only on the behavior of the boundary of $S$ in the neighborhood of $z=\infty$, so it is no essential loss of generality to assume, as we do, the relation $\min \theta(u)>\max \phi_{+}(u)$ for all $u$ for which $\theta(u)$ is defined.

Denote by $S^{*}$ the infinite strip in the $w$-plane which contains $S$ and whose boundaries are contained in the loci $v= \pm \theta(u)$. The locus $v= \pm \theta(u)$, where all allowable values of $\phi_{+}(u)$ and $\phi_{-}(u)$ are admitted in the equation $\theta(u)$ $\equiv \phi_{+}(u)-\phi_{-}(u)$, may separate the plane into even an infinite number of regions. One such region contains $S$ and is denoted by $S^{*}$. Let $\Sigma_{\zeta}$ in the $\zeta(=\xi+i \eta)$-plane be the strip $|\eta|<\pi / 2$, and let $w=g(\zeta)$ map $\Sigma_{\zeta}$ onto $S^{*}$ so that $\xi=+\infty$ corresponds to $u=+\infty$. Denote by $\gamma_{+}$and $\gamma_{-}$the loci $\eta=\eta_{+}(\xi)$ 
and $\eta=\eta_{-}(\xi)$, namely the respective images in the $\zeta$-plane of the loci $v=\phi_{+}(u)$ and $v=\phi_{-}(u)$. By Theorem 2.3 we have

$$
\lim _{\xi \rightarrow \infty} \eta_{+}(\xi)=\frac{1}{1-\lambda} \frac{\pi}{2}, \quad \lim _{\xi \rightarrow \infty} \eta_{-}(\xi)=\frac{\lambda}{1-\lambda} \frac{\pi}{2} .
$$

Let $\Omega_{\zeta}$ denote the image of $S$ in the $\zeta$-plane under the map $w=g(\zeta)$, and let finally

$$
z=-\log (1-s), \quad s=\sigma+i \tau,
$$

where $z$ is real when $s$ is real, and

$$
\zeta=-\log (1-t)
$$

where $\zeta$ is real when $t$ is real. The transformation (3.3) maps $\Sigma_{z}$ onto the half-plane $\sigma<1$, and it follows from (3.2) that (3.4) maps $\Omega_{\zeta}$ onto a region in the $t$-plane whose boundary has an angle $\pi / 2$ at $t=1$ and which contains some interval $t_{1}<t<1, t_{1}<1$.

Let $x_{n}$ be an arbitrary sequence of real points in the z-plane with $x_{n} \rightarrow+\infty$, and set $s_{n}=s\left(x_{n}\right), w_{n}=f\left(x_{n}\right), \zeta_{n}=\xi_{n}+i \eta_{n}=\zeta\left(w_{n}\right), t_{n}=t\left(\zeta_{n}\right)$. It is well known (Lindelöf) that in the transformation from the $t$-plane to the $s$-plane angles are transformed proportionally, so we have from (3.4) and (3.2)

$$
\lim _{n \rightarrow \infty} \eta_{n}=-\lim _{n \rightarrow \infty} \arg \left(1-t_{n}\right)=\frac{1+\lambda}{1-\lambda} \frac{\pi}{4} .
$$

From (3.5) and Theorem 2.3, we have

$$
\lim _{n \rightarrow \infty} \frac{v\left(\zeta_{n}\right)}{\theta\left[u\left(\zeta_{n}\right)\right]}=\frac{1}{2} \frac{1+\lambda}{1-\lambda},
$$

which with a slight change of notation is written

$$
\lim _{n \rightarrow \infty} \frac{v\left(x_{n}\right)}{\theta\left[u\left(x_{n}\right)\right]}=\frac{1}{2} \frac{1+\lambda}{1-\lambda} .
$$

We proceed further using the method of proof of Theorem 2.1, by setting here

$$
W=U+i V=f_{n}(z) \equiv \frac{f\left(z+x_{n}\right)-f\left(x_{n}\right)}{\theta\left[u\left(x_{n}\right)\right]} ;
$$

the denominator here is not the same as in (2.2); moreover in (2.2) the number $f\left(x_{n}\right)$ is real but not necessarily so in (3.7); in (2.2) we have $f_{n}^{\prime}(0)>0$ but not necessarily in (3.7). We write

$$
\frac{d w}{d z}=\frac{d w}{d \zeta} \cdot \frac{d \zeta}{d t} \cdot \frac{d t}{d s} \cdot \frac{d s}{d z}=\dot{g}^{\prime}(\zeta)(1-t)^{-1} t^{\prime}(s) e^{-z},
$$




$$
\arg f^{\prime}\left(x_{n}\right)=\arg g^{\prime}\left(\zeta_{n}\right)-\arg \left(1-t_{n}\right)+\arg t^{\prime}\left(s_{n}\right) .
$$

Theorem 2.1 applies to the map $w=g(\zeta)$, so from differentiation of (2.1) we have arg $g^{\prime}\left(\zeta_{n}\right) \rightarrow 0$. From this fact and Visser's formula (see for instance $\left[2\right.$, equation (8) ]) we deduce by (3.8) that arg $f^{\prime}\left(x_{n}\right) \rightarrow 0$. Then by (3.6) the image of $S$ under the sequence of transformations (3.7) consists of a sequence of regions converging to a kernel, precisely the infinite strip $|V|<1 / 2$. Equation (3.1) follows by the Carathéodory theory.

We treat now the case $\lambda=0$, hitherto excluded. From the assumption $\phi_{-}(u) / \phi_{+}(u) \rightarrow 0$ it follows that for each $u$ sufficiently large we have min $\phi_{+}(u)$ $>\max \left[-\phi_{-}(u)\right]$; since the asymptotic behavior of $f(z)$ as $x \rightarrow \infty$ depends only on the behavior of the boundary of $S$ in the neighborhood of infinity, it is no loss of generality for us to assume, as we do, $\min \phi_{+}(u)>\max \left[-\phi_{-}(u)\right]$ for all $u$ for which these functions are defined. As $u \rightarrow \infty$ we have $\phi_{+}(u) / \theta(u)$ $\rightarrow 1$, so in order to prove (3.1) it is sufficient to prove

$$
\lim _{n \rightarrow \infty} \frac{f\left(z+x_{n}\right)-f\left(x_{n}\right)}{\phi_{+}\left[u\left(x_{n}\right)\right]}=\frac{z}{\pi} ;
$$

the function $\phi_{+}(u)$ has property $B$, by Theorem 1.4; the method already used now applies without essential change, where $\phi_{+}(u)$ takes the role previously taken by $\theta(u)$, and this completes the proof of Theorem 3.1.

A strip bounded by two loci $v=\phi_{+}(u)$ and $v=\phi_{-}(u)<\phi_{+}(u)$ is called an $L$-strip if each of these loci has an $L$-tangent at infinity.

When $S$ is an $L$-strip, the real part of (3.1) is given by Warschawski [4, (18.7)]. Indeed, for this case (3.1) can be readily proved by his methods. If $z_{1}=x_{1}+i y_{1}, z_{2}=x_{2}+i y_{2}, x_{2}>x_{1}$, we have

$$
f\left(z_{2}\right)-f\left(z_{1}\right)=\int_{z_{1}}^{z_{2}} f^{\prime}(z) d z
$$

where the path of integration is a line segment. By [4, Theorem II] if $\left|z-z_{1}\right| \leqq M$,

$$
f^{\prime}(z) / f^{\prime}\left(z_{1}\right) \rightarrow 1 \quad \text { as } \quad x_{1} \rightarrow+\infty,
$$

uniformly for $\left|y_{1}\right|,|y| \leqq \beta<\pi / 2$. But by [4, Theorem $\mathrm{X}$ (ii)]

$$
f^{\prime}\left(z_{1}\right)=(1 / \pi) \theta\left[u\left(x_{1}\right)\right][1+o(1)] \text { as } x_{1} \rightarrow+\infty,
$$

uniformly for $\left|y_{1}\right| \leqq \beta$. Combination of these relations yields

$$
\frac{f\left(z_{2}\right)-f\left(z_{1}\right)}{\theta\left[u\left(x_{1}\right)\right]}=\frac{z_{2}-z_{1}}{\pi}+o(1)
$$

uniformly for $\left|z_{1}-z_{2}\right| \leqq M,\left|y_{1}\right| \leqq \beta$. Equation (3.1) follows with $z_{-}=x_{n}$ $z_{2}=x+x_{n}$. 
Theorem 3.1 has applications to the further study of the behavior of the mapping function.

Theorem 3.2. With the hypothesis of Theorem 3.1 let $z_{n}=x_{n}+i y_{n}$ be a sequence of points of $\Sigma_{z}$ such that $x_{n} \rightarrow+\infty, \lim \sup \left|y_{n}\right|<\pi / 2$. Then we have

$$
\begin{aligned}
& \lim _{n \rightarrow \infty} \frac{f^{\prime}\left(z_{n}\right)}{\theta\left[u\left(z_{n}\right)\right]}=\frac{1}{\pi}, \\
& \lim _{n \rightarrow \infty} \frac{f^{(v)}\left(z_{n}\right)}{\theta\left[u\left(z_{n}\right)\right]}=0, \\
& \lim _{n \rightarrow \infty} \arg f^{\prime}\left(z_{n}\right)=0 .
\end{aligned}
$$

Equations (1) and (3) were proved by Warschawski [4, pp. 315, 288] with the stronger hypothesis that $S$ is an $L$-strip, but without our restrictions $\phi_{-}(u) / \phi_{+}(u) \rightarrow \lambda$ and that a segment $u_{1}<u<+\infty, v=0$ lie in $S$. He proves (3) uniformly in $|y|<\pi / 2$ without the restriction lim sup $\left|y_{n}\right|<\pi / 2$.

We differentiate the two members of (3.1) and then set $z=i y_{n}$; this set of points is contained in a closed bounded subset of $S$, so we have

$$
\lim _{n \rightarrow \infty} \frac{f^{\prime}\left(z_{n}\right)}{\theta\left[u\left(x_{n}\right)\right]}=\frac{1}{\pi} .
$$

Equation (3.1) yields

$$
\frac{u\left(z_{n}\right)-u\left(x_{n}\right)}{\theta\left[u\left(x_{n}\right)\right]} \rightarrow 0,
$$

from which Theorem 1.2 and (3.9) yield (1). Equation (3) follows at once from (1), and (2) follows from (3.1) by repeated differentiation.

We leave to the reader a proof similar to that of Theorem 3.2 which establishes

Theorem 3.3. With the hypothesis of Theorem 3.1, let $z_{n}=x_{n}+i y_{n}, z_{n}^{\prime}=x_{n}^{\prime}$ $+i y_{n}^{\prime}$ be two sequences of points of $\Sigma_{z}$ such that

$$
x_{n} \rightarrow+\infty
$$

with $\lim \sup _{n \rightarrow \infty}\left|x_{n}-x_{n}^{\prime}\right|<+\infty, \lim \sup _{n \rightarrow \infty}\left|y_{n}\right|<\pi / 2, \lim \sup _{n \rightarrow \infty}\left|y_{n}^{\prime}\right|$ $<\pi / 2$. Then we have

$$
\lim _{n \rightarrow \infty}\left[\frac{u\left(z_{n}\right)-u\left(z_{n}^{\prime}\right)}{\theta\left[u\left(z_{n}\right)\right]}-\frac{x_{n}-x_{n}^{\prime}}{\pi}\right]=0 ;
$$

If $\lim _{n \rightarrow \infty} y_{n}=\beta,|\beta|<\pi / 2$, then

$$
\lim _{n \rightarrow \infty} \frac{v\left(z_{n}\right)}{\theta\left[u\left(z_{n}\right)\right]}=\frac{1}{2} \frac{1+\lambda}{1-\lambda}+\frac{\beta}{\pi} .
$$


Part (1) is proved by Warschawski $[4$, p. 324] and part (2) also by Warschawski $[4$, p. 315]; he supposes that $S$ is an $L$-strip and proves (2) uniformly for $|\beta|<\pi / 2$, without some of our restrictions.

4. Some extensions. We proceed to prove an extension of Theorem 3.1:

THEOREM 4.1. Theorem 3.1 remains valid if we replace $\lim \phi_{-}(u) / \phi_{+}(u)$ $=\lambda$ by the condition

$$
0<a \leqq\left|\phi_{-}(u) / \phi_{+}(u)\right| \leqq b<+\infty
$$

and require that both $\phi_{+}(u)$ and $\phi_{-}(u)$ have property $\mathrm{B}$.

It follows here by Theorem 1.6 that $\theta(u) \equiv \phi_{+}(u)-\phi_{-}(u)$ has property $\mathrm{B} \cdot$ For each $u$ sufficiently large, we have $\min \theta(u)>\max \phi_{+}(u)$. Indeed, in the equation

$$
\frac{\min \theta(u)}{\max \phi_{+}(u)}=\frac{\min \phi_{+}(u)}{\max \phi_{+}(u)}-\frac{\min \left[-\phi_{-}(\mathrm{u})\right]}{\max \phi_{+}(u)},
$$

the first term of the second member approaches unity as $u$ becomes infinite and the second term is not less than $a(>0)$ for all $u$. As in the proof of Theorem 3.1 we assume $\min \theta(u)>\max \phi_{+}(u), \min \theta(u)>-\min \phi_{-}(u)$ for all $u$, which involves no essential loss of generality.

We proceed to prove Theorem 4.1 in several steps, by successive specializations of the region $S$.

LemMA 1. Theorem 4.1 is true if $\phi_{+}(u) \equiv \pi / 2$.

The function $\theta(u) \equiv \pi / 2-\phi_{-}(u)$ has property $\mathrm{B}$, by Theorem 1.6. Then we can apply Theorem 2.1 to the function $w^{\prime}=u^{\prime}+i v^{\prime}=g\left(z^{\prime}\right), z^{\prime}=x^{\prime}+i y^{\prime}$, which maps the strip $\left|y^{\prime}\right|<\pi$ onto the symmetric strip of the $w^{\prime}$-plane whose boundary consists of the loci $v^{\prime}= \pm \theta\left(u^{\prime}\right)$. Lemma 1 follows by combining the transformations $w^{\prime}=g\left(z^{\prime}\right), w^{\prime}=w-i \pi / 2, z^{\prime}=z-i \pi / 2$.

Lemma 2. With the hypothesis of Theorem 2.1 let $\gamma_{\sigma}$ be a curve in $S$ defined for $u_{1}<u<+\infty$ by the equation $v=\sigma(u)$, and suppose $\sigma(u)$ to have property $\mathrm{B}$. Suppose $\lim \inf _{u \rightarrow+\infty}|\sigma(u) / \theta(u)|>0$ and $\lim \sup _{u \rightarrow \infty}|\sigma(u) / \theta(u)|<1 / 2$, with $\theta(u) \equiv 2 \phi(u)$. Let $\Gamma_{\sigma}$ be the image in the z-plane of $\gamma_{\sigma}$ under the mapping $w=f(z)$, represented by $y=y_{\sigma}(x)$. Then $y_{\sigma}(x)$ has property B at $x=+\infty$.

It follows from Theorem 2.3 that the ordinates of $\Gamma_{\sigma}$ are in absolute value bounded from zero and $\pi / 2$, hence follows from (2.1) that we have

$$
\lim _{n \rightarrow \infty}\left[\frac{\sigma(u)}{\theta(u)}-\frac{y_{\sigma}}{\pi}\right]=0 .
$$

Our hypothesis on $\lim \inf |\sigma(u) / \theta(u)|$ now yields 


$$
\lim _{x \rightarrow \infty} \frac{y_{\sigma}(x)}{\pi} / \frac{\sigma(u)}{\theta(u)}=1 .
$$

Let $I_{0}$ be the interval $|X|<X_{0}$, where $X_{0}$ is arbitrary. Set $x^{\prime}=X y_{\sigma}(x)+x$, $u^{\prime}=u\left[x^{\prime}+i y_{\sigma}\left(x^{\prime}\right)\right]$. By the method of proof of (4.1) we have

$$
\lim _{x \rightarrow \infty} \frac{y_{\sigma}\left(x^{\prime}\right)}{\pi} / \frac{\sigma\left(u^{\prime}\right)}{\theta\left(u^{\prime}\right)}=1
$$

uniformly for $X$ in $I_{0}$. By virtue of (4.1) and (4.2) it is now sufficient for the proof of the lemma to show

$$
\lim _{x \rightarrow \infty} \frac{\sigma(u)}{\sigma\left(u^{\prime}\right)}=\lim _{x \rightarrow \infty} \frac{\theta(u)}{\theta\left(u^{\prime}\right)}=1,
$$

uniformly for $X$ in $I_{0}$.

By Theorem 2.2 we have

$$
\lim _{n \rightarrow \infty}\left[\frac{u^{\prime}-u}{\theta(u)}-\frac{X y_{\sigma}(x)}{\pi}\right]=0 .
$$

Since $X$ is in $I_{0}$ and $\left|y_{\sigma}(x)\right|<\pi / 2$, we conclude by Theorem 1.1

$$
\lim _{x \rightarrow \infty} \frac{\theta(u)}{\theta\left(u^{\prime}\right)}=1 \text {, }
$$

the second part of (4.3). Equation (4.4) suggests the form

$$
u^{\prime}=M(x) \frac{\theta(u)}{\sigma(u)} \sigma(u)+u ;
$$

since $M(x)$ is uniformly bounded and $\lim \inf |\sigma(u) / \theta(u)|>0$, and since $\sigma(u)$ has property $B$ at $+\infty$ the first part of (4.3) follows. Lemma 2 is established.

Lemмa 3. With the hypothesis of Lemma 1 let $x_{n}$ be any sequence of real points with $\lim _{n \rightarrow \infty} x_{n}=+\infty$. Then with the notation $\psi(u) \equiv\left[\phi_{-}(u)-\pi / 2\right] / 2$ we have

$$
\lim _{n \rightarrow \infty} \frac{v\left(x_{n}\right)-\psi\left[u\left(x_{n}\right)\right]}{\theta\left[u\left(x_{n}\right)\right]}=0 .
$$

This lemma follows in a manner analogous to the derivation of (3.6).

Lemma 4. If $\phi_{+}(u)$ and $\phi_{-}(u)$ satisfy the hypothesis of Theorem 4.1 and also the inequalities $0<a_{1} \leqq \phi_{+}(u) \leqq a_{2}<\pi / 2, a_{1} \leqq-\phi_{-}(u) \leqq a_{2}$ for $u_{1}<u<+\infty$, and if $\lim _{u \rightarrow+\infty} \theta(u)=\pi / 2$, then the conclusion of Theorem 4.1 is valid.

Let $S^{\prime}$ be the strip of the $w$-plane bounded by the two loci $v=\phi_{-}(u)$, $v=\pi / 2, u_{1}<u<+\infty$, and let $w=g(\zeta), \zeta=\xi+i \eta$ map the strip $S_{\zeta}:|\eta|<\pi / 2$ 
onto $S^{\prime}$ so that $w=+\infty$ corresponds to $\zeta=+\infty$. Let $\eta=\eta_{+}(\xi)$ denote the image of the curve $v=\phi_{+}(u)$ under the inverse of the mapping $w=g(\zeta)$; from Lemmas 1 and 3 with the notation $\psi_{0}(u) \equiv \pi / 2-\phi_{-}[u(\xi)]$, we have

$$
\begin{aligned}
\lim _{\xi \rightarrow+\infty}\left[\frac{\phi_{+}[u(\xi)]}{\psi_{0}(u)}-\frac{1}{2} \frac{\pi / 2+\phi_{-}[u(\xi)]}{\psi_{0}(u)}-\frac{\eta_{+}(\xi)}{\pi}\right] & =0, \\
\lim _{\xi \rightarrow+\infty}\left[\frac{\phi_{+}[u(\xi)]}{\psi_{0}(u)}-\frac{2 \eta_{+}(\xi)}{\pi}\right] & =0 .
\end{aligned}
$$

From the inequality $\phi_{+}[u(\xi)] \geqq a_{1}>0$ it follows by the method of proof of Lemma 2 that $\eta_{+}(\xi)$ has property B.

Let $\zeta=h(z)$ map $S_{z}$ onto the strip of the $\zeta$-plane bounded by $\eta_{+}(\xi)$ and $\eta=-\pi / 2$, by the composition $g(\zeta) \equiv g[h(z)] \equiv f(z)$. For any sequence of real points $x_{n}, x_{n} \rightarrow+\infty$, we have from Lemma 1

$$
\lim _{n \rightarrow \infty} \frac{h\left(z+x_{n}\right)-h\left(x_{n}\right)}{\pi / 2+\eta_{+}\left[\xi\left(x_{n}\right)\right]}=\frac{z}{\pi}
$$

throughout $S_{z}$, uniformly on any closed bounded subset of $S_{z}$. Likewise for any sequence of real points $\xi_{n}, \xi_{n} \rightarrow \infty$, we have

$$
\lim _{n \rightarrow \infty} \frac{g\left(\zeta+\xi_{n}\right)-g\left(\xi_{n}\right)}{\psi_{0}\left[u\left(\xi_{n}\right)\right]}=\frac{\zeta}{\pi}
$$

throughout $S_{\zeta}$, uniformly on any closed bounded subset of $S_{\zeta}$.

With the notation $\zeta_{n}=h\left(x_{n}\right)=\xi_{n}+i \eta_{n}, \zeta_{n}^{\prime}=h\left(z+x_{n}\right)=\xi_{n}{ }^{\prime}+i \eta_{n}{ }^{\prime}$, we have from (4.6) applied to $\zeta=h(z)$ the relation $\lim \sup _{n \rightarrow \infty}\left|\xi_{n}^{\prime}-\xi_{n}\right|<\infty$, whence from (4.7)

$$
\begin{aligned}
& \lim _{n \rightarrow \infty} {\left[\frac{g\left(\xi_{n}^{\prime}\right)-g\left(\xi_{n}\right)}{\psi_{0}\left[u\left(\xi_{n}\right)\right]}-\frac{\xi_{n}^{\prime}-\xi_{n}}{\pi}\right]=0, } \\
& \lim _{u \rightarrow \infty}\left[\frac{g\left(\zeta_{n}\right)-g\left(\xi_{n}\right)}{\psi_{0}\left[u\left(\xi_{n}\right)\right]}-\frac{i \eta_{n}}{\pi}\right]=0, \\
& \lim _{n \rightarrow \infty}\left[\frac{g\left(\zeta_{n}^{\prime}\right)-g\left(\xi_{n}^{\prime}\right)}{\psi_{0}\left[u\left(\xi_{n}^{\prime}\right)\right]}-\frac{i \eta_{n}^{\prime}}{\pi}\right]=0 .
\end{aligned}
$$

Theorem 1.2 enables us to replace $\psi_{0}\left[u\left(\xi_{n}^{\prime}\right)\right]$ in (4.10) by $\psi_{0}\left[u\left(\xi_{n}\right)\right]$, so by (4.8), (4.9), (4.10) we obtain

$$
\lim _{n \rightarrow \infty}\left[\frac{g\left(\zeta_{n}^{\prime}\right)-g\left(\zeta_{n}\right)}{\psi_{0}\left[u\left(\xi_{n}\right)\right]}-\frac{\zeta_{n}^{\prime}-\zeta_{n}}{\pi}\right]=0 .
$$

Again by Theorem 1.2 we can here replace $\psi_{0}\left[u\left(\xi_{n}\right)\right]$ by $\psi_{0}\left[u\left(\zeta_{n}\right)\right]$. Thus from 
(4.11), (4.5), and (4.6) with $\theta(u) \rightarrow \pi / 2$ we have

$$
\lim _{n \rightarrow \infty}\left[f\left(z+x_{n}\right)-f\left(x_{n}\right)\right]=z / 2
$$

throughout $S_{z}$, and the uniformity on any closed bounded subset of $S_{z}$ follows readily. This completes the proof of Lemma 4.

We are now in a position to complete the proof of Theorem 4.1. Let $S^{\prime}$ be the symmetric strip in the $w$-plane whose boundary is the loci $v= \pm \theta(u)$, $u_{1}<u<+\infty$. It follows from Theorem 1.6 that $\theta(u)$ has property $\mathrm{B}$. We use the notation $w=g(\zeta), \zeta=\xi+i \eta$ for the function which maps $S_{\zeta}:|\eta|<\pi / 2$ onto $S^{\prime}$ such that $\xi=+\infty$ corresponds to $u=+\infty$. If $\xi_{n}$ is any sequence of real points with $\xi_{n} \rightarrow+\infty$ as $n \rightarrow \infty$, we have from Theorem 2.1

$$
\lim _{n \rightarrow \infty}\left[\frac{g\left(\zeta+\xi_{n}\right)-g\left(\xi_{n}\right)}{\theta\left[u\left(\xi_{n}\right)\right]}\right]=\frac{2 \zeta}{\pi}
$$

throughout $S_{5}$, uniformly on any closed bounded subset of $S_{\zeta}$.

By Lemma 2 the images of $v=\phi_{-}(u)$ and $v=\phi_{+}(u)$ under the inverse of the mapping $w=g(\zeta)$ are curves $\eta=\eta_{-}(\xi)$ and $\eta=\eta_{+}(\xi)$ which have property B. Moreover both of the latter curves satisfy the additional hypothesis of Lemma 4. For each $\xi$ we set $\zeta_{+}=\xi+i \eta_{+}(\xi), \zeta_{-}=\xi+i \eta_{-}(\xi)$, whence

$$
\begin{aligned}
& \lim _{\xi \rightarrow+\infty}\left[\frac{\phi_{+}\left[u\left(\zeta_{+}\right)\right]}{\theta[u(\xi)]}-\frac{2 \eta_{+}(\xi)}{\pi}\right]=0, \\
& \lim _{\xi \rightarrow+\infty}\left[\frac{\phi_{-}\left[u\left(\zeta_{-}\right)\right]}{\theta[u(\xi)]}-\frac{2 \eta_{-}(\xi)}{\pi}\right]=0 .
\end{aligned}
$$

From (4.12) it is readily shown that

$$
\lim _{\xi \rightarrow+\infty}\left[\frac{u\left(\zeta_{+}\right)-u(\xi)}{\theta[(u(\xi)]}\right]=0,
$$

whence by Theorem 1.1

$$
\lim _{\xi \rightarrow+\infty}\left[\frac{\phi_{+}\left[u\left(\zeta_{+}\right)\right]-\phi_{-}\left[u\left(\zeta_{-}\right)\right]}{\theta[u(\xi)]}\right]=1 .
$$

Equations (4.15) and (4.13) yield

$$
\lim _{\xi \rightarrow+\infty}\left[\eta_{+}(\xi)-\eta_{-}(\xi)\right]=\pi / 2 .
$$

We now define the mapping $\zeta=h(z)$ by the equations $g(\zeta) \equiv g[h(z)] \equiv f(z)$. If $x_{n}$ is any sequence of real points of $S_{z}$ with $x_{n} \rightarrow+\infty$ as $n \rightarrow \infty$, we have by (4.16) and Lemma 4

$$
\lim _{n \rightarrow \infty}\left[h\left(z+x_{n}\right)-h\left(x_{n}\right)\right]=z / 2
$$


throughout $S_{z}$, uniformly on any closed bounded subset of $S_{z}$. By combining (4.12) and (4.17) as in the proof of Lemma 4 we have (3.1) throughout $S_{z}$, uniformly on any closed bounded subset of $S_{z}$, which establishes Theorem 4.1.

It is apparent that Theorems 3.2 and 3.3, proved from Theorem 3.1, have analogues similarly proved from Theorem 4.1 :

THEOREM 4.2. With the hypothesis of Theorem 4.1 let $z_{n}=x_{n}+i y_{n}$ be a sequence of points of $S_{z}$ with $x_{n} \rightarrow+\infty, \lim \sup _{n \rightarrow \infty}\left|y_{n}\right|<\pi / 2$. Then we have

$$
\begin{array}{ll}
\lim _{n \rightarrow \infty} \frac{f^{\prime}\left(z_{n}\right)}{\theta\left[u\left(z_{n}\right)\right]}=\frac{1}{\pi}, & \\
\lim _{n \rightarrow \infty} \frac{f^{(\nu)}\left(z_{n}\right)}{\theta\left[u\left(z_{n}\right)\right]}=0, & \nu=2,3, \cdots, \\
\lim _{n \rightarrow \infty} \arg f^{\prime}\left(z_{n}\right) & =0 .
\end{array}
$$

THEOREM 4.3. With the hypothesis of Theorem 4.1 let $z_{n}=x_{n}+i y_{n}$, $z_{n}^{\prime}=x_{n}^{\prime}+i y_{n}^{\prime}$ be two sequences of points of $S_{z}$ with $x_{n} \rightarrow+\infty, x_{n}^{\prime} \rightarrow+\infty$, $\lim \sup \left|x_{n}-x_{n}^{\prime}\right|<\infty, \lim \sup \left|y_{n}\right|<\pi / 2, \lim \sup \left|y_{n}^{\prime}\right|<\pi / 2$. Then

$$
\lim _{n \rightarrow \infty}\left[\frac{u\left(z_{n}^{\prime}\right)-u\left(z_{n}\right)}{\theta\left[u\left(z_{n}\right)\right]}-\frac{x_{n}^{\prime}-x_{n}}{\pi}\right]=0 .
$$

If $\lim _{n \rightarrow \infty} y_{n}=\beta,|\beta|<\pi / 2$, then

$$
\lim _{n \rightarrow \infty} \frac{v\left(z_{n}\right)-v\left(x_{n}\right)}{\theta\left[u\left(z_{n}\right)\right]}=\frac{\beta}{\pi} .
$$

Parts (1) and (3) of Theorem 4.2 were obtained by Warschawski [4, pp. $315,288]$ for the case that $S$ is an $L$-strip but without our restriction on $\left|\phi_{-}(u) / \phi_{+}(u)\right|$. Warschawski does not assume lim sup $\left|y_{n}\right|<\pi / 2$ nor that $S$ contains the segment $u>u_{1}, v=0$. Likewise part (1) of Theorem 4.3 was obtained by Warschawski $[4$, p. 324] when $S$ is an $L$-strip without our restriction on lim sup $\left|y_{n}\right|$ and lim sup $\left|y_{n}^{\prime}\right|$. Part (2) of Theorem 4.3 was obtained by Warschawski for $L$-strips $[4,(17.10)]$.

From Theorems 4.2 and 4.3 we shall obtain also

ThEOREM 4.4. With the hypothesis of Theorem 4.3 we have

$$
\begin{aligned}
\lim _{n \rightarrow \infty} \theta\left[u\left(z_{n}\right)\right] / \theta\left[u\left(z_{n}^{\prime}\right)\right] & =1, \\
\lim _{n \rightarrow \infty} f^{\prime}\left(z_{n}\right) / f^{\prime}\left(z_{n}^{\prime}\right) & =1 .
\end{aligned}
$$

Part (1) is a consequence of part (1) of Theorem 4.3 and Theorem 1.2; part (2) is a consequence of part (1) and part (1) of Theorem 4.2. Warschawski [4, p. 288] proved part (2) for an $L$-strip. 
5. A further extension. Thus far we have envisaged only strips $S$ containing an infinite segment $u_{1}<u<+\infty$ of the axis of reals. We now remove that restriction.

THEOREM 5.1. Let $S$ be a strip in the plane of $w=u+i v$ whose boundary consists of the two loci $v=\phi_{+}(u), v=\phi_{-}(u),-\infty<u_{1}<u<+\infty$, where $\phi_{+}(u)$ is positive and has property B. Let $w=f(z), z=x+i y$, map the strip $S_{z}:|y|<\pi / 2$ onto $S$ such that $u(z) \rightarrow+\infty$ when $x \rightarrow+\infty$. If $x_{n}$ is any sequence of real points with $x_{n} \rightarrow+\infty$, and if $\lim _{u \rightarrow+\infty} \phi_{-}(u) / \phi_{+}(u)=\lambda, 0 \leqq \lambda<1$, then

$$
\lim _{n \rightarrow \infty} \frac{f\left(z+x_{n}\right)-f\left(x_{n}\right)}{\theta\left[u\left(x_{n}\right)\right]}=\frac{z}{\pi}
$$

for $z$ in $S_{z}$, uniformly on any closed bounded subset of $S_{z}$, where $\theta(u) \equiv \phi_{+}(u)$ $-\phi_{-}(u)$.

We shall not give a detailed proof, for the technique is essentially the same as that used in the proof of Theorem 3.1. Analogous supplementary conditions are imposed, such as $\phi_{+}(u) \geqq\left|\phi_{-}(u)\right|$ for $u_{1}<u<+\infty$. The proof is based on the ability to replace $S$ by a symmetric region containing $S$ to which we can apply the results of the previous sections.

Let $w=g(\zeta), \zeta=\xi+i \eta$, map the strip $S_{\zeta}:|\eta|<\pi / 2$ onto the strip $S^{\prime}$ whose boundary consists of the two loci $v= \pm \phi_{+}(u), u_{1}<u<+\infty$. Theorem 2.1 applies to the mapping function $g(\zeta)$. By Theorem 2.3 and Theorem 1.6'the image of $S$ under the inverse of the mapping $w=g(\zeta)$ is a region in the $\zeta$-plane, which is transformed by the vertical translation $\zeta^{\prime}=\zeta-(1+\lambda) \pi i / 4$ into a region satisfying the hypothesis of Theorem 3.1. The composite mapping can then be treated by the methods already used, and the theorem follows.

Applications of Theorem 5.1 analogous to those of Theorem 3.1 are

Theorem 5.2. With the hypothesis of Theorem 5.1 let $z_{n}=x_{n}+i y_{n}$ be a sequence of points of $S_{z}$ such that $x_{n} \rightarrow+\infty$ and $\lim \sup _{n \rightarrow \infty}\left|y_{n}\right|<\pi / 2$. Then we have

$$
\begin{aligned}
& \lim _{n \rightarrow \infty} \frac{f^{\prime}\left(z_{n}\right)}{\theta\left[u\left(z_{n}\right)\right]}=\frac{1}{\pi}, \\
& \lim _{n \rightarrow \infty} \frac{f^{(\nu)}\left(z_{n}\right)}{\theta\left[u\left(z_{n}\right)\right]}=0, \\
& \lim _{n \rightarrow \infty} \arg f^{\prime}\left(z_{n}\right)=0 .
\end{aligned}
$$

THEOREM 5.3. With the hypothesis of Theorem 5.1, let $z_{n}=x_{n}+i y_{n}$ and $z_{n}^{\prime}=x_{n}^{\prime}+i y_{n}^{\prime}$ be two sequences of points of $S_{z}$ with $x_{n} \rightarrow+\infty, x_{n}^{\prime} \rightarrow+\infty$, $\lim \sup \left|x_{n}-x_{n}^{\prime}\right|<+\infty, \lim \sup _{n \rightarrow \infty}\left|y_{n}\right|<\pi / 2$, lim sup $\left|y_{n}^{\prime}\right|<\pi / 2$. Then we have 


$$
\lim _{n \rightarrow \infty}\left[\frac{u\left(z_{n}\right)-u\left(z_{n}{ }^{\prime}\right)}{\theta\left[u\left(z_{n}\right)\right\rfloor}-\frac{x_{n}-x_{n}^{\prime}}{\pi}\right]=0 ;
$$

if $\lim _{n \rightarrow \infty} y_{n}=\beta,|\beta|<\pi / 2$, then

$$
\lim _{n \rightarrow \infty} \frac{v\left(z_{n}\right)}{\theta\left[u\left(z_{n}\right)\right]}=\frac{1}{2} \frac{1+\lambda}{1-\lambda}+\frac{\beta}{\pi} .
$$

The relation of Theorem 3.1 to Theorem 4.1 corresponds to the relation of Theorem 5.1 to

THEOREM 5.4. The conclusion of Theorem 5.1 is valid if $\phi_{-}(u)$ has property $\mathrm{B}$ and the condition $\lim _{u \rightarrow \infty} \phi_{-}(u) / \phi_{+}(u)=\lambda$ is replaced by

$$
0<a_{1} \leqq\left|\phi_{-}(u) / \phi_{+}(u)\right| \leqq a_{2}<1 .
$$

Analogues of Theorems 5.2 and 5.3 are

Theorem 5.5. With the hypothesis of Theorem 5.4, let $z_{n}=x_{n}+i y_{n}$ be a sequence of points of $S_{z}$ such that $x_{n} \rightarrow \infty, \lim \sup \left|y_{n}\right|<\pi / 2$. Then the conclusion of Theorem 4.2 is valid.

Theorem 5.6. If $S$ satisfies the conditions of Theorem 5.4 rather than those of Theorem 4.1, the conclusions of Theorems 4.3 and 4.4 are valid.

Our previous references to Warschawski apply also to the present section.

6. Zero angles at a finite point. We consider in this section the analogues of our previous results, but where the significant boundary points are finite rather than at infinity. We choose rather to apply our previous results than the methods used to prove them, but this choice is merely a matter of convenience.

A function $\phi(u)$ defined in the interval $u_{1}<u \leqq 1\left(-\infty \leqq u_{1}<1\right)$ is said to have property $\mathrm{B}$ at $u=1$ if $\phi(u) \rightarrow 0$ as $u \rightarrow 1^{-}$and if uniformly in any interval $I:|U| \leqq U_{0}$ we have

$$
\lim _{u \rightarrow 1^{-}} \frac{\phi[U \phi(u)+u]}{\phi(u)}=1 .
$$

We do not require that $\phi(u)$ be single valued, but do require that (6.1) hold for any choice of values of $\phi(u)$.

It is obvious that only a slight modification of the proofs of $\$ 1$ yields results on functions having property $\mathrm{B}$ at $u=1$ analogous to those of $\S 1$. For instance we have $\phi(u) /(1-u) \rightarrow 0$ as $u \rightarrow 1$. It is perhaps more important that property $B$ is invariant under inversion:

Theorem 6.1. Suppose the function $y=\phi(x)$ has property B at $x=1$. Set

$$
x=1-u /\left(u^{2}+v^{2}\right), y=v /\left(u^{2}+v^{2}\right) .
$$

Then the function $v=h(u)$ defined by $y=\phi(x)$ and (6.2) has property B at 
$u=+\infty$. Conversely, if $v=\Phi(u)$ has property B at $u=+\infty$, then the function $y=H(x)$ defined by $v=\Phi(u)$ and (6.2) has property B at $x=1$.

We omit the proof of Theorem 6.1, which is relatively straightforward and depends on the results of $\$ 1$ and their analogues.

An immediate consequence of Theorems 6.1 and 3.1 is

THEOREM 6.2. Let $w=f(z)$ with $w=u+i v, z=x+i y$ map the half plane $R_{z}: x<1$ onto the simply connected region $R$ of the w-plane which contains the interval $u_{1}<u<1$ but not the point at infinity, with $f(1)=1$. Suppose that the boundary of $R$ consists of two loci $v=\phi_{+}(u), v=\phi_{-}(u)$ for $u_{2}<u<1$, that $\phi_{+}(u)>0$ and $\phi_{-}(u)<0$ for $u_{2}<u<1$, and that $\phi_{+}(u)$ has property B at $u=1$. Suppose also $\lim _{u \rightarrow 1}-\phi_{-}(u) / \phi_{+}(u)=\lambda,-\infty<\lambda \leqq 0$. Then if $0<x_{n}<1$ is any sequence of real points with $\lim _{n \rightarrow \infty} x_{n}=1$, we have

$$
\lim _{n \rightarrow \infty} \frac{f\left[\left(1-x_{n}\right) z+x_{n}\right]-f\left(x_{n}\right)}{\theta\left[u\left(x_{n}\right)\right]}=-\frac{1}{\pi} \log (1-z)
$$

throughout $R_{z}$, uniformly on any closed bounded subset of $R_{z}$, where $\theta(u) \equiv \phi_{+}(u)$ $-\phi_{-}(u)$.

Both for Theorem 6.2 and Theorem 6.6 below we assume min $\theta(u)$ $>\left\{\max \phi_{+}(u)-\min \phi_{-}(u)\right\}$ for each $u$ for which $\theta(u)$ is defined. This assumption involves no essential loss of generality, as is indicated for property B at $u=+\infty$ instead of $u=1^{-}$in connection with Theorems 3.1 and 4.1.

An immediate consequence of Theorem 6.2 is

THEOREM 6.3. With the hypothesis of Theorem 6.2 let $z_{n}$ and $z_{n}^{\prime}$ be two sequences of points of $R_{z}$ sucin that $z_{n} \rightarrow 1$ and $z_{n}^{\prime} \rightarrow 1$ "in angle," namely so that $\lim \sup _{n \rightarrow \infty}\left|\arg \left(1-z_{n}\right)\right|<\pi / 2$, lim $\sup _{n \rightarrow \infty}\left|\arg \left(1-z_{n}^{\prime}\right)\right|<\pi / 2$, and also so that we have $0<c_{1} \leqq\left|\left(1-z_{n}\right) /\left(1-z_{n}^{\prime}\right)\right| \leqq c_{2}<\infty$. Then we have

$$
\begin{aligned}
& \lim _{n \rightarrow \infty} \frac{1-f\left(z_{n}\right)}{1-f\left(z_{n}^{\prime}\right)}=1, \\
& \lim _{n \rightarrow \infty} \frac{f^{\prime}\left(z_{n}\right)\left(1-z_{n}\right)}{f^{\prime}\left(z_{n}^{\prime}\right)\left(1-z_{n}^{\prime}\right)}=1 .
\end{aligned}
$$

Since $z_{n}=x_{n}+i y_{n} \rightarrow 1$ in angle, the set of points $i y_{n} /\left(1-x_{n}\right)$ is contained in a closed bounded subset of $R_{z}$. By (6.3) we obtain

$$
\lim _{n \rightarrow \infty}\left[\frac{f\left(z_{n}\right)-f\left(z_{n}\right)}{\theta\left[u\left(x_{n}\right)\right]}+\frac{1}{\pi} \log \frac{1-z_{n}}{1-x_{n}}\right]=0 ;
$$

the analogue of Theorem 1.3 is $\phi(u) /(1-u) \rightarrow 0$, from which we have

$$
\lim _{n \rightarrow \infty} \frac{1-f\left(z_{n}\right)}{1-f\left(x_{n}\right)}=1 \text {. }
$$


In a similar manner we have

$$
\lim _{n \rightarrow \infty} \frac{1-f\left(z_{n}^{\prime}\right)}{1-f\left(x_{n}^{\prime}\right)}=1 .
$$

From the last part of the hypothesis it follows that the sequence

$$
\left(x_{n}^{\prime}-x_{n}\right) /\left(1-x_{n}\right)
$$

lies in a closed bounded subset of $R_{z}$; thus we write

$$
\begin{gathered}
\lim _{n \rightarrow \infty}\left[\frac{f\left(x_{n}^{\prime}\right)-f\left(x_{n}\right)}{\theta\left[u\left(x_{n}\right)\right]}+\frac{1}{\pi} \log \frac{1-x_{n}^{\prime}}{1-x_{n}}\right]=0, \\
\lim _{n \rightarrow \infty} \frac{1-f\left(x_{n}\right)}{1-f\left(x_{n}^{\prime}\right)}=1 .
\end{gathered}
$$

Part (1) now follows immediately. To obtain part (2) we differentiate (6.3) and obtain

$$
\lim _{n \rightarrow \infty} \frac{\left(1-x_{n}\right)(1-z) f^{\prime}\left[\left(1-x_{n}\right) z+x_{n}\right]}{\theta\left[u\left(x_{n}\right)\right]}=\frac{1}{\pi},
$$

uniformly on any closed bounded subset of $R_{z}$. By the method used in part (1) and from the relation $\theta\left[u\left(z_{n}\right)\right] / \theta\left[u\left(z_{n}^{\prime}\right)\right] \rightarrow 1$ we deduce part (2) from (6.4).

Ostrowski [5, p. 176] proved part (1) of Theorem 6.3 with the weaker hypothesis that the boundary of $R$ forms a zero angle at the accessible point $w=1$. He proved part (2) without the "in angle" restriction provided the boundary curves have an $L$-tangent at $w=1$, and under those conditions showed that the "in angle" restriction can be removed from part (1).

Similar methods can be used to establish

TheOREM 6.4. With the hypothesis of Theorem 6.2 let $z_{n}$ be a sequence of points of $R_{z}$ such that $z_{n} \rightarrow 1$ in angle. Then we have

$$
\begin{aligned}
\lim _{n \rightarrow \infty}\left[\arg f^{\prime}\left(z_{n}\right)+\arg \left(1-z_{n}\right)\right] & =0, & \\
& \lim _{n \rightarrow \infty} \inf \frac{\log \left|f^{\prime}\left(z_{n}\right)\right|}{\log \left|1-z_{n}\right|} \geqq-1, & \\
\lim _{n \rightarrow \infty} \frac{f^{(\nu)}\left(z_{n}\right)\left(1-z_{n}{ }^{\nu}\right)}{\theta\left[u\left(z_{n}\right)\right]} & =\frac{(\nu-1) !}{\pi}, & \nu=1,2, \cdots, \\
\lim _{n \rightarrow \infty} \frac{f^{(\nu)}\left(z_{n}\right)\left(1-z_{n}\right)^{\nu}}{1-f\left(z_{n}\right)} & =0, & \nu=1,2, \cdots, \\
\lim _{n \rightarrow \infty} \frac{f^{(\nu+1)}\left(z_{n}\right)\left(1-z_{n}\right)^{\nu}}{f^{\prime}\left(z_{n}\right)} & =\nu !, & \nu=0,1, \cdots .
\end{aligned}
$$


Ostrowski proved parts (1), (2) [5, p. 184] and (5) [5, p. 130] for the case that the boundary of $R$ has an $L$-tangent at $w=1$, without the "in angle" assumption for part (1) and with equality for part (2). He also proved (4) $[5$, p. 130] under the same assumptions as he used for part (1) of Theorem 6.3.

Theorem 6.5 is another immediate consequence of Theorem 6.2 ; the conclusion is an analogue for the zero angle case of the Lindelöf theorem on proportionality of angles. Theorem 6.5 is stronger in conclusion but more restrictive in hypothesis than the corresponding result due to Warschawski $[4$, p. 327].

THEOREM 6.5. With the hypothesis of Theorem 6.3 and the added conditions $\arg \left(1-z_{n}\right) \rightarrow \alpha,|\alpha|<\pi / 2, \arg \left(1-z_{n}^{\prime}\right) \rightarrow \beta,|\beta|<\pi / 2, \beta \neq \pi(1+\lambda) / 2(1-\lambda)$, we have

$$
\lim _{n \rightarrow \infty} \frac{\arg \left[1-f\left(z_{n}\right)\right]}{\arg \left[1-f\left(z_{n}^{\prime}\right)\right]}=\frac{2(1-\lambda) \alpha-\pi(1+\lambda)}{2(1-\lambda) \beta-\pi(1+\lambda)} .
$$

We can modify Theorem 6.2 by applying Theorem 4.1 instead of Theorem 3.1:

THEOREM 6.6. Theorem 6.2 remains true if the condition

$$
\lim _{u \rightarrow 1} \frac{\phi_{-}(u)}{\phi_{+}(u)}=\lambda
$$

is replaced by the inequalities $0<a \leqq\left|\phi_{-}(u) / \phi_{+}(u)\right| \leqq b<\infty$ and the condition that $\phi_{-}(u)$ and $\phi_{+}(u)$ have property $\mathrm{B}$ at $u=1$.

Theorems 6.3 and 6.4 likewise can be modified by replacing Theorem 6.2 by Theorem 6.6 in the hypothesis of each.

Theorem 5.1 has an analogue, which we proceed to formulate.

We say that a simply connected region $R$ of the w-plane is admissible if either of the following sets of conditions is satisfied:

(1) the boundary of $R$ consists of the two loci $v=\phi_{+}(u), v=\phi_{-}(u)$, $-\infty \leqq u_{1}<u<1$.

(2) the function $\phi_{+}(u)$ is positive $u_{1}<u<1$.

(3) $\phi_{+}(u)$ has property $\mathrm{B}$ at $u=1$.

(4) $\lim _{u \rightarrow 1^{-}} \phi_{-}(u) / \phi_{+}(u)=\lambda(0 \leqq \lambda<1)$.

$\left(3^{\prime}\right) \phi_{-}(u)$ and $\phi_{+}(u)$ have property $\mathrm{B}$ at $u=1$.

$\left(4^{\prime}\right) \lim \inf _{u \rightarrow 1-\phi_{-}}(u) / \phi_{+}(u)>0$, lim $\sup _{u \rightarrow 1}-\phi_{-}(u) / \phi_{+}(u)<1$.

Use of 6.1 in conjunction with Theorems 5.1 and 5.4 yields

TheOREM 6.7. Let $w=f(z)$ map the half plane $R_{z}: x<1$ onto the admissible region $R$ such that $z \rightarrow 1$ implies $f(z) \rightarrow 1$. Then if $x_{n}$ is any sequence of real points with $0<x_{n}<1, x_{n} \rightarrow 1$, we have 


$$
\lim _{n \rightarrow \infty} \frac{f\left[\left(1-x_{n}\right) z+x_{n}\right]-f\left(x_{n}\right)}{\theta\left[u\left(x_{n}\right)\right]}=-\frac{1}{\pi} \log (1-z)
$$

throughout $R_{z}$, uniformly on any closed bounded subset of $R_{z}$, where $\theta(u) \equiv \phi_{+}(u)$ $-\phi_{-}(u)$.

THEOREM 6.8. With the hypothesis of Theorem 6.7 let $z_{n}, z_{n}{ }^{\prime}$ be two sequences of points of $R_{z}$ with $z_{n} \rightarrow 1$ and $z_{n}^{\prime} \rightarrow 1$ in angle, and $0<c_{1} \leqq\left|\left(1-z_{n}\right) /\left(1-z_{n}^{\prime}\right)\right|$ $\leqq c_{2}<\infty$. Then we have

$$
\begin{aligned}
\lim _{n \rightarrow \infty} \frac{1-f\left(z_{n}\right)}{1-f\left(z_{n}^{\prime}\right)} & =1, \\
\lim _{n \rightarrow \infty} \frac{f^{\prime}\left(z_{n}\right)\left(1-z_{n}\right)}{f^{\prime}\left(z_{n}^{\prime}\right)\left(1-z_{n}^{\prime}\right)} & =1 .
\end{aligned}
$$

THEOREM 6.9. With the hypothesis of Theorem 6.7 let $z_{n}$ be a sequence of points of $R_{z}$ such that $z_{n} \rightarrow 1$ in angle. Then we have (1), (2), (3), (4), (5) of Theorem 6.4 .

7. Conditions for convergence to kernel. We have based the previous discussion ( $\$ \S 1-5)$ on condition (1.1) rather than on the condition

$$
\lim _{u_{n} \rightarrow \infty} \frac{\phi\left[U \phi\left(u_{n}\right)+u_{n}\right]}{\phi\left(u_{n}\right)}=1
$$

uniformly for $U$ in every interval $I$; this is of course merely a matter of choice, corresponding to the limit of the continuous variable $u$ rather than the limit of a sequence $u_{n}$. Condition (7.1) might be termed property $\mathrm{B}$ with respect to a particular sequence $u_{n}$; it is obviously the more discriminating condition, and can be made fundamental in our treatment instead of (1.1). Results thus obtained are wholly analogous to those already formulated in detail.

As an illustration here (compare [1]), let the strip $S$ consist of the circles $|w-n|<1 / 4(n=0,1,2, \cdots)$ joined by horizontal canals of respective widths $1 / 8,1 / 16, \cdots$, constant for each canal, the entire strip $S$ being symmetric in the $u$-axis. If the points $u_{n}$ in (7.1) are chosen as the midpoints of the canals, the kernel of the regions $S_{n}$ (notation of $\$ 2$ ) consists of the strip $|V|<1$; here $(2.1)$ is valid, where $u_{n}=f\left(x_{n}\right)$. If the points $u_{n}$ in (7.1) are chosen as the points $n=0,1,2, \cdots, \phi\left(u_{n}\right)=1 / 4$, the kernel of the regions $S_{n}$ consists of the circle $|W|<1$; here the second member of $(2.1)$ is to be replaced by the function which maps $\Sigma$ onto $|W|<1$ with $z=0$ corresponding to $W=0$ and with directions at those points invariant.

Neither (1.1) nor (7.1) implies the existence of an $L$-tangent; indeed, given a smooth curve $v=\phi(u)>0$ with an $L$-tangent we may modify $\phi(u)$ by placing a countable infinity of small vertical cuts extending downward from the curve 
at the abscissas $u=1,2, \cdots$, or at the points $u_{n}$ if the latter are given. The length of each cut divided by the original ordinate at the same abscissa shall approach zero as the (variable) abscissa becomes infinite. Then the new ordinate $\phi_{1}(u)$ has property $B$; the new ordinate is in part multiple valued, and the locus $v=\phi_{1}(u)$ does not possess an $L$-tangent. The existence of an $L$-tangent is frequently required by Ostrowski and Warschawski.

In the special case that $\phi(u)$ or $\phi\left(u_{n}\right)$ is bounded and bounded from zero, (1.1) and (7.1) can be written in the respective forms

$$
\begin{aligned}
\phi[U+u]-\phi(u) & \rightarrow 0, \\
\phi\left[U+u_{n}\right]-\phi\left(u_{n}\right) & \rightarrow 0,
\end{aligned}
$$

uniformly for $U$ in every interval $I$. It is thus clear by the mean value theorem that if $\phi^{\prime}(u)$ exists for every $u$ and approaches zero as $u \rightarrow \infty$, these conditions (7.2) and (7.3) are satisfied. The condition that $\phi^{\prime}(u)$ exists and approaches zero of course implies that $S$ has an horizontal $L$-tangent at infinity. Under these circumstances the regions $S_{n}$ (we assume for simplicity $S$ to be symmetric in the $u$-axis) converge to a kernel which is an infinite strip bounded by two lines parallel to and equidistant from the $U$-axis; equation (2.1) can be written in the form

$$
\lim _{n \rightarrow \infty}\left[f\left(z+x_{n}\right)-f\left(x_{n}\right)\right] / \phi\left[u\left(x_{n}\right)\right]=\lambda z,
$$

where the constant $\lambda$ is suitably chosen.

However, compare Madame Lelong-Ferrand [3, \$13], it is not sufficient for this conclusion that (7.3) hold nonuniformly with respect to $U$ in $I$, as we indicate by a counter-example.

We denote by $\psi_{k}(u)$ the continuous even function which takes the values $0,1-1 / k, 0,0$, in the respective points $u= \pm 1, u= \pm(1+1 / 2 k)$, $u= \pm(1+1 / k), \pm \infty$, and which is linear in the successive intervals of $-\infty<u<+\infty$ bounded by these points. We note the relation $\psi_{k}(u) \rightarrow 0$ as $k \rightarrow \infty$ for every $u$. We now define

$$
\phi(u) \equiv 1-\sum_{k=1}^{\infty} \psi_{k}\left(u-2^{k+1}\right) ;
$$

of course this infinite series contains for each $u$ at most one nonzero term. The graph of the function $\phi(u)$ is the profile of a saw of infinite length whose teeth become sharper and approach unity in height as the distance from the origin becomes infinite. We set here $u_{n}=2^{n}$, whence

$$
\phi\left(u_{n}+U\right)-\phi\left(u_{n}\right) \equiv \sum_{k=1}^{\infty} \psi_{k}\left(2^{n}-2^{k+1}\right)-\sum_{k=1}^{\infty} \psi_{k}\left(U+2^{n}-2^{k+1}\right),
$$

so that (7.1) and (7.3) are satisfied for every $U$ but not uniformly for $U$ in every interval $I$. We have $\phi\left(u_{n}\right)=1$, and if the region $S$ is bounded by the loci 
$v= \pm \phi(u)$, the regions $S_{n}$ (notation of $\S 2$ ) converge to a kernel which consists of the square $|U|<1,|V|<1$. Equation (2.1) is not valid but becomes valid if the second member of (2.1) is replaced by the function which maps $\Sigma$ onto this kernel so that the origins correspond to each other and directions at the origins are invariant.

Condition (7.1) holding uniformly is sufficient but not necessary that the regions $S_{n}$ converge to the kernel $|V|<1$, and hence that (2.1) be valid; this is shown by the counter-example of a region $S$ bounded by the loci $v= \pm \phi(u)$,

$$
\phi(u) \equiv 1+\sum_{k=1}^{\infty} \psi_{k}\left(u-2^{k+1}\right)
$$

where $\psi_{k}$ is defined above; we have $\left(U_{0}>1\right)$

$$
\lim _{n \rightarrow \infty}\left\{\max \left[\phi\left(U+u_{n}\right),|U| \leqq U_{0}\right]\right\}=2 .
$$

Nevertheless a necessary condition that regions $S_{n}$ found as in $\$ 2$ from a region $S$ bounded by $v= \pm \phi(u)$ converge to the kernel $|V|<1$ is for every $U_{0}(>0)$

$$
\lim _{u_{n} \rightarrow \infty} \frac{\min \left\{\phi\left[U \phi\left(u_{n}\right)+u_{n}\right],|U| \leqq U_{0}\right\}}{\phi\left(u_{n}\right)}=1 ;
$$

for $n$ sufficiently large all the $S_{n}$ must contain the region $|V|<1-\delta,|U|<U_{0}$ where $\delta(>0)$ is arbitrary; for $n$ sufficiently large all the $S_{n}$ can contain no region $|V|<1+\delta,|U|<U_{0}, \delta>0$. This necessary condition combined with the condition

$$
\lim _{u_{n} \rightarrow \infty} \frac{\phi\left[U \phi\left(u_{n}\right)+u_{n}\right]}{\phi\left(u_{n}\right)}=1
$$

for a set of values $U$ everywhere dense on $-\infty<U<+\infty$ is sufficient that the regions $S_{n}$ found from a region $S$ bounded by $v= \pm \phi(u)$ converge to the kernel $|V|<1$.

A necessary and sufficient condition for (2.1), if $S$ is a region bounded by $v= \pm \phi(u)$, is that the regions $S_{n}$ converge to the kernel $|V|<1$. Of course, condition (7.3) without uniformity is not necessary for the $S_{n}$ to converge to the kernel $|V|<1$ in the case $\phi\left(u_{n}\right) \rightarrow 1$, as is shown by the counter-example (7.4).

Condition (7.1) is readily expressed in terms of a mean value of $\phi^{\prime}(u)$, provided $\phi^{\prime}(u)$ exists and $\phi(u)$ can be expressed as an indefinite integral of $\phi^{\prime}(u)$. We have

$$
\frac{\phi\left[U \phi\left(u_{n}\right)+u_{n}\right]-\phi\left(u_{n}\right)}{\phi\left(u_{n}\right)}=\frac{1}{\phi\left(u_{n}\right)} \int_{u_{n}}^{u_{n}+U \phi\left(u_{n}\right)} \phi^{\prime}(u) d u,
$$


so the condition

$$
\lim _{n \rightarrow \infty} \frac{1}{\phi\left(u_{n}\right)} \int_{u_{n}}^{u_{n}+U \phi\left(u_{n}\right)} \phi^{\prime}(u) d u=0
$$

uniformly for $U$ in every $I$ is sufficient for (7.1). Of course (7.5) is satisfied if the mean value relation

$$
\lim _{n \rightarrow \infty} \frac{1}{U \phi\left(u_{n}\right)} \int_{u_{n}}^{u_{n}+U \phi\left(u_{n}\right)} \phi^{\prime}(u) d u=0
$$

is satisfied uniformly for $U$ in every $I$.

Madame Lelong-Ferrand points out that if $\int^{\infty}\left[\phi^{\prime}(u)\right]^{2} d u$ exists, we may write

$$
\left[\phi\left(u_{n}+l\right)-\phi\left(u_{n}\right)\right]^{2}<l \int_{u_{n}}^{u_{n}+l}\left[\phi^{\prime}(u)\right]^{2} d u ;
$$

this last member approaches zero uniformly for bounded $l$ as $u_{n} \rightarrow \infty$, so (7.3) is satisfied uniformly for $U$ in $I$. If in addition $\lim \inf \phi\left(u_{n}\right)>0$, condition (7.1) is satisfied uniformly for $U$ in $I$.

\section{BIBLIOGRAPHY}

1. C. Carathéodory, Conformal representation, Cambridge University Press, 1932.

2. J. L. Walsh, On distortion at the boundary of a conformal map, Proc. Nat. Acad. Sci. U.S.A. vol. 36 (1950) pp. 152-156.

3. J. Lelong-Ferrand, Sur la représentation conforme des bandes, Journal d'Analyse Mathématique vol. 2 (1952) pp. 51-71.

4. S. E. Warschawski, On conformal mapping of infinite strips, Trans. Amer. Math.Soc. vol. 51 (1942) pp. 280-335.

5. A. Ostrowski, Über den Habitus der konformen Abbildung am Rande des Abbildungsbereiches, Acta Math. vol. 64 (1935) pp. 81-184.

6. C. Gattegno and A. Ostrowski, Représentation conforme à la frontière: domaines généraux; domaines particuliers, Mémorial des Sci. Math., nos. 109 and 110, Paris, 1949.

7. J. Ferrand, Etude de la représentation conforme au voisinage de la frontière, Ann. École Norm. (3) vol. 59 (1942) pp. 43-106.

8. —, Nouvelle demonstration d'un théorème de M. Ostrowski, C.R. Acad. Sci. Paris vol. 220 (1945) pp. 550-551.

HARVARD UNIVERSITY,

Cambridge, Mass.

Melpar, InC., Cambridge, Mass. 